\title{
Philosophiques
}

\section{La métaphore heuristique de l'esclavage dans les textes féministes}

\section{Guy Bouchard}

Volume 14, numéro 1, printemps 1987

URI : https://id.erudit.org/iderudit/027004ar

DOI : https://doi.org/10.7202/027004ar

Aller au sommaire du numéro

Éditeur(s)

Société de philosophie du Québec

ISSN

0316-2923 (imprimé)

1492-1391 (numérique)

Découvrir la revue

Citer cet article

Bouchard, G. (1987). La métaphore heuristique de l'esclavage dans les textes féministes. Philosophiques, 14(1), 112-144. https://doi.org/10.7202/027004ar
Résumé de l'article

Conjoignant théorie de la métaphore et conception hétéro-politique de l'utopie, cet article montre comment la métaphore de l'esclavage, dans les textes féministes, constitue un instrument heuristique nous incitant à redéfinir à la fois nos sociétés et nos théories. d'utilisation que vous pouvez consulter en ligne.

https://apropos.erudit.org/fr/usagers/politique-dutilisation/ 


\title{
LA MÉTAPHORE HEURISTIQUE DE L'ESCLAVAGE DANS LES TEXTES FÉMINISTES *
}

\author{
par Guy Bouchard
}

\begin{abstract}
RÉSUMÉ. Conjoignant théorie de la métaphore et conception hétéropolitique de l'utopie, cet article montre comment la métaphore de l'esclavage, dans les textes féministes, constitue un instrument heuristique nous incitant à redéfinir à la fois nos sociétés et nos théories.
\end{abstract}

ABSTRACT. Combining the theory of the metaphor and an heteropolitical view of utopia, this paper shows how the metaphor of slavery, in feminist discourses, constitutes an heuristic device that incites us to redefine our societies as well as our theories.

De la métaphore à l'utopie: ce parcours conjoindra deux déplacements. Définie par Aristote comme transport ${ }^{1}$, la métaphore mime, sur le plan linguistique, le passage d'un lieu à un autre. Toujours située ailleurs qu'ici et maintenant, l'utopie entraîne le lecteur dans un autre lieu, dans un temps différent, voire dans un nouvel espace relevant simultanément d'une autre perspective temporelle.

De la métaphore à l'utopie: ce double déplacement sollicitera une métaphore particulière, celle de l'esclavage dans les textes féministes, dont le sens profond, débordant la simple

* Texte rédigé dans le cadre d'un projet subventionné par le Conseil de recherches en sciences humaines du Canada, et consacré à la philosophie hétéropolitique du féminisme. Une version abrégée de ce texte a été présentée au colloque Parole (Université de Sherbrooke, 13-15 mars 1986).

1. "La métaphore est le transport à une chose d'un nom qui en désigne une autre, transport du genre à l'espèce ou de l'espèce au genre ou de l'espèce à l'espèce ou d'après le rapport d'analogie" (1921 : 1457 1. 6.9). 
description analogique, émerge dans un monde utopique, conjure une société hétéropolitique. Le fait de langage devient facteur historiogène.

\section{LES RÔLES DE LA MÉTAPHORE ${ }^{2}$}

Dans La métaphore vive, Ricœur (1975) oppose la conception dite substitutive de la métaphore à une théorie soidisant interactive. D'Aristote au Groupe de Liège, en passant par Du Marsais, Fontanier, Ullmann et Konrad, entre autres, la conception substitutive élaborée par la rhétorique classique et raffinée par son prolongement structuraliste réduirait la métaphore à un phénomène sémiotique, c'est-à-dire à un accident de la dénomination jouant de l'écart entre sens propre et sens figuré, ainsi que de la substitution du terme emprunté au terme propre, pour concocter un colifichet verbal dénué de toute valeur informative. La métaphore vive par contre, celle qui intéresse des théoriciens comme Richards, Black et Beardsley, serait plutôt un énoncé caractérisé par une prédication impertinente sollicitant une nouvelle pertinence fondée sur une ressemblance transcatégoriale; et ce serait grâce à cette transgression catégoriale que la métaphore procéderait à une redescription heuristique de la réalité, redescription essentielle non seulement à la démarche scientifique, mais aussi au déploiement de la pensée philosophique.

Cette opposition entre la métaphore-mot et la métaphoreénoncé m'apparaît arbitraire. Considérons par exemple la définition de Fontanier, présentée par Ricour comme le paradigme de la rhétorique classique. À première vue, dans la mesure où la métaphore est un trope et où le trope est une figure en un seul mot (1968: 66-67, 73, 99), on pourrait croire que, pour Fontanier, la métaphore n'est qu'une histoire de termes. Mais lisons mieux. La métaphore est un trope. Or les tropes sont des «sens plus ou moins différents du sens primitif, qu'offrent, dans l'expression de la pensée, les mots appliqués à de nouvelles idées " (1968: 39). Mais si «l'expression de la pensée par la parole se compose de mots » (1968: 41) et s'articule en proposition (1968: 51 , il faut comprendre que les tropes, et partant la métaphore,

2. Pour l'ensemble de cette section, cf. Bouchard 1984. 
sont des sens différents qu'offrent, dans la proposition, les mots appliqués à de nouvelles idées. Autrement dit, pour la rhétorique classique, aucun mot n'est en lui-même métaphorique, le transport métaphorique ne circule que dans l'énoncé : thèse que confirmeraient, au besoin, tous les exemples de métaphore de tous les rhétoriqueurs. Inversement, lorsqu'une théorie interactive, en l'occurrence celle de Max Black (1979), présente la métaphore comme une phrase ou une expression du même genre dans laquelle certains mots sont employés métaphoriquement et d'autres non métaphoriquement, et lorsqu'elle propose, pour expliquer l'interaction entre le foyer métaphorique et les autres mots de l'énoncé, le recours à un «système de lieux communs associés », ne met-elle pas en scène les mêmes acteurs que la conception précédente?

Je plaide donc pour la complémentarité des conceptions dites substitutive et interactive. Qui met d'abord l'accent sur le mot en relation avec une autre expression qu'il remplace, admet pourtant que cette substitution a lieu dans l'énoncé. Qui attire l'attention sur l'énoncé, reconnaît pourtant que ce sont certains mots qui sont employés métaphoriquement, qu'il met en rapport avec d'autres sens associés. Cette complémentarité est celle du paradigme et du syntagme, nécessaire à la compréhension du fonctionnement métaphorique comme de toute utilisation du langage.

Le langage a ses règles. Le respect absolu de celles-ci le transformerait en masse inerte, incapable de s'adapter à la nouveauté du monde et à l'humeur fluctuante de ses utilisateurs. La transgression de toutes les règles aboutirait à la dérive absolue et à l'incommunicabilité. Toute figure, tout trope contrevient à quelque règle, mais selon un protocole. Au niveau sémantique intraphrastique, le dérèglement de tous les sens se traduit par une incompatibilité entre termes co-présents. Le protocole tropique est une matrice permettant à l'interprète d'extirper, de l'absurdité apparente, un sens inattendu. Si vous établissez un rapport d'inclusion entre le terme déviant et un terme virtuel : synecdoque. Un rapport de contiguité : métonymie. Un rapport de ressemblance: métaphore. En termes techniques :

1) La métaphore est une expression linguistique appartenant à la classe des expressions caractérisées par la notion d'écart, à savoir, 
pour reprendre la terminologie du Groupe de Liège, les métaboles.

2) L'écart qui caractérise la métaphore se situe au niveau sémantique, plus précisément au niveau des métasémèmes.

3) La métaphore comporte un aspect syntagmatique et un aspect paradigmatique.

4) L'aspect syntagmatique met en cause au minimum deux signes $A$ et $B$, composés chacun d'un signifiant et d'un signifié, et dont l'un, le modificateur (A) dans les termes de Beardsley, modifie l'autre, le terme modifié $(\mathbf{B})$.

5) L'aspect paradigmatique met en cause un consigne composé d'un consignifiant et d'un consignifié, et substituable au modificateur.

6) L'écart métaphorique joue à deux niveaux: d'une part entre modificateur et consigne, d'autre part entre modificateur et terme modifié ; autrement dit, l'écart est à la fois paradigmatique et syntagmatique, il introduit une double tension : association figurée d'une part, impertinence sémantique d'autre part.

7) Le fondement de la métaphore est une ressemblance qui, conjointe à une inclusion ou à une exclusion, réduit l'écart en faisant voir, par l'entremise du consigne, sous quels aspects le terme modifié est comme le terme modificateur, et inversement (interaction des éléments de la métaphore).

«Bref, la métaphore est un métasémène qui d'une part, sur le plan syntagmatique, combine un modificateur et un terme modifié en tension d'impertinence sémantique et d'autre part, sur le plan paradigmatique, associe le modificateur à un consigne en tension figurée, tension que la ressemblance entre le signifié du modificateur et le consignifié permet de réduire au profit d'une nouvelle pertinence interactive ». (Bouchard 1984: 119-120)

«L'homme est un loup-garou». Au sens littéral, la fausseté d'un tel énoncé est évidente. Pour lui faire rendre sens, j'explorerai le champ sémique des deux termes jusqu'à ce que je leur découvre une classe commune expliquant leur ressemblance : celle par exemple, de l'humain masculin qui, métamorphosé, devient féroce. La métaphore est sphinx et, comme tous les sphinx, elle engendre un laïus, un parcours interprétatif plus ou moins complexe jouant, dans la communication humaine, une multiplicité de rôles.

Dans La métaphore vive, Ricour insiste sur le rôle cognitif de la métaphore, sur sa fonction de redescription heuristique de la réalité, neutralisant une référence de premier degré pour déployer une référence de second degré liée au monde de l'œuvre. Cela l'incite à contester la thèse de la non-référentialité du 
discours littéraire, à introduire la notion de vérité métaphorique, puis à marquer les différences qui, malgré tout, continuent de séparer discours spéculatif et discours poétique. Cette entreprise, si louable qu'elle puisse apparaître à l'encontre des théories réduisant la métaphore à un simple rôle ornemental, et la littérature à une complaisance envers le non-sens, engendre cependant une série de problèmes redoutables. Prenons par exemple la notion de référence dédoublée appliquée aux œuvres d'art. Décider que la dénotation de l'œuvre constitue le «monde » de l'œuvre, n'est-ce pas simplement jouer d'une métaphore? Soit la référence au sens courant, c'est-à-dire le renvoi au (à $l e$ ) monde; soit en plus la référence au sens second, c'est-à-dire le renvoi au «monde» de l'œuvre; soit enfin la référence au sens élargi, englobant les deux cas précédents et renvoyant aux mondes: prétendre que l'œuvre a une référence (au sens élargi) parce qu'elle a une référence seconde ne lui conférera jamais une référence au sens courant. Concevoir l'absence de dénotation comme une dénotation de degré zéro, cela n'est sans doute pas plus instructif que n'est enrichissante la conception de la pauvreté comme degré zéro de la richesse!

Le problème, en fait, est mal posé. Lorsque Ricœur déclare que la métaphore n'est pas un ornement du discours mais que, comportant une information nouvelle, elle dit quelque chose de nouveau sur la réalité, il présente comme incompatibles deux fonctions qui, chez Aristote déjà, étaient conjointes. La métaphore, dit Aristote, fait l'ornement du style, mais elle enseigne aussi par le genre; aliment et assaisonnement fréquentent la même assiette. Et si l'on écoute aussi Cicéron, et Quintilien, et Augustin, et Du Marsais, et Fontanier, et Blair, et Whately, entre autres, on se fera quelque idée de la polyvalence de la métaphore: suppléer aux carences du vocabulaire, pallier les incapacités du terme propre, donner de l'éclat et du relief au discours, le rendre plus concis, l'ennoblir, le rendre plus énergique, témoigner de la créativité de l'orateur, faire interagir les idées, faire voir l'invisible, présenter l'inanimé comme agissant, déguiser les idées qu'on n'ose exprimer directement, exprimer pensées et sentiments, s'adapter aux capacités intellectuelles du destinaire, rendre la vérité consommable. Sans doute certains de ces rôles sont-ils regroupables. On pourrait, à 
cette fin, utiliser les six fonctions du langage proposées par Jakobson, les fonctions poétique, référentielle, émotive, métalinguistique, conative et phatique. Mais pourquoi ces fonctions ne pourraient-elles s'associer deux à deux, ou trois à trois, ou quatre à quatre, ou cinq à cinq, voire réaliser une coalition hexagonale? Il n'y a pas lieu, on le voit, de choisir entre fonction ornementale et fonction cognitive : non seulement les deux ne sont pas incompatibles, mais encore d'autres fonctions sont possibles, qui témoignent de la plasticité de la métaphore et de la multiplicité des intérêts de ceux et de celles qui dérèglent le langage pour dire l'imprévu.

Relativiser de la sorte la fonction cognitive de la métaphore, ce n'est pas minimiser son importance. Dans certains types de discours, selon certaines stratégies, le recours à une métaphore peut projeter un éclairage tout à fait neuf sur un domaine encore mal exploré ou au contraire considéré comme parfaitement élucidé. Tel est le cas de la métaphore de l'esclavage.

\section{LA MÉTAPHORE DE L'ESCLAVAGE DANS LES TEXTES FÉMINISTES}

\subsection{D’Aristote à Simone de Beauvoir...}

L'origine naturelle de la cité remonte, selon Aristote (1962: I, 1252a23-1256b40), à la première union nécessaire, qui est celle de deux êtres incapables d'exister l'un sans l'autre, comme «c'est le cas pour le mâle et la femelle en vue de la procréation »; comme c'est encore le cas «de l'union de celui dont la nature est de commander avec celui dont la nature est d'être commandé, en vue de leur conservation commune ». Ainsi donc, «c'est la nature qui a distingué la femelle et l'esclave ». L'homme, la femelle, l'esclave : voilà constituée la famille, et du regroupement de plusieurs familles naît le village, et du rassemblement de quelques villages procède la cité. Mais « dans les rapports du mâle et de la femelle, le mâle est par nature supérieur, et la femelle inférieure, et le premier est l'élément dominateur et la seconde l'élément dominé ». Ce n'est certes pas par hasard qu'Aristote discute ainsi les rapports entre épouse et époux dans le contexte de sa théorie de l'esclavage. Une métaphore inédite hante ce texte qui, en se 
contentant d'en juxtaposer les éléments, la programme pour les siècles à venir : car si le rapport de l'homme à la femme est celui du maître à l'esclave, alors, selon le protocole même de la métaphore analogique, qui permet d'employer le quatrième terme à la place du second et le second au lieu du quatrième (Aristote 1961 : 1457b16-19), la femme est l'esclave de l'homme, et l'épouse d'un esclave est esclave de l'esclave.

Mais si les esclavagistes du sexe féminin ne pouvaient pas, sans se démasquer, énoncer cette métaphore, les antiesclavagistes l'ont proclamée depuis les débuts du féminisme. Mary Wollstonecraft (1976: 200), par exemple :

Le devoir qu'on attend d'elles, comme tous les devoirs imposés arbitrairement aux femmes, provient plus d'un sens des convenances et du respect des convenances que de la raison; et apprenant ainsi à se soumettre servilement à leurs parents, elles se préparent à l'esclavage du mariage.

Flora Tristan (in Michaud 1984: 124-125):

Mais, prolétaires, il vous reste à vous, hommes de 1843 , une cuvre non moins grande à accomplir: à votre tour, affranchissez les dernières esclaves qui restent encore dans la société française, proclamez les DROITS DE LA FEMME [...]

John Stuart Mill (1975: 97-98, 164):

Je suis loin de prétendre que les femmes ne sont pas en général mieux traitées que les esclaves. Mais aucun esclave n'est esclave à un tel point et dans un sens aussi fort du terme qu'une femme [...]

Le mariage est le seul véritable esclavage reconnu par notre loi. Légalement il ne reste plus d'esclave sinon la maîtresse de chaque maison.

Friedrich Engels (1966: 71-72):

La famille conjugale moderne est fondée sur l'esclavage domestique, avoué ou voilé, de la femme, et la société moderne est une masse qui se compose exclusivement de familles conjugales, comme d'autant de molécules.

Auguste Bebel (1979: 9-10):

La femme est le premier être bumain qui ait eu à éprouver la servitude. Elle a été esclave avant même que l'esclave fût. 
Simone de Beauvoir (1968A : 22, 189; 1968B : 378) :

Or la femme a toujours été, sinon l'esclave de l'homme, du moins sa vassale $[\ldots]$

Assimiler la femme à l'esclave est une erreur; il y a eu parmi les esclaves des femmes, mais il a toujours existé des femmes libres [...]

[La femme amoureuse] choisit de vouloir si ardemment son esclavage qu'il lui apparaîtra comme l'expression de sa liberté [...]

Ces exemples ont été choisis parmi les plus illustres. Tant dans une perspective diachronique que dans un survol des textes féministes contemporains, on pourrait les multiplier indéfiniment. La métaphore de l'esclavage est la métaphore féministe par excellence. Du strict point de vue de la fonction ornementale, son omniprésence pourrait relever du cliché. Mais il ne s'agit pas de stylistique. Sa récurrence même, et le type de textes qu'elle hante, suggèrent d'autres rôles. Un rôle heuristique, par exemple.

Sur le plan paradigmatique, la métaphore associe, nous l'avons souligné, un modificateur à un consigne. Pour la rhétorique classique, le consigne était la signification littérale substituable $^{3}$ à la signification métaphorique. Dans une conception comme celle de Max Black, il s'agit plutôt d'une pluralité de lieux communs associés. Or, loin d'être incompatibles, ces deux théories sont complémentaires, la première constituant un cas-limite de la seconde. Et l'application systématique d'un consigne pluriel à un terme modifié peut nous permettre d'envisager celui-ci dans une nouvelle perspective.

Selon les dictionnaires courants, le mot « esclavage » renvoie d'abord à l'état, à la condition de l'esclave, c'est-à-dire à une personne qui est sous la puissance absolue d'un maître, comme le furent les esclaves de l'antiquité grecque et latine, et les Noirs jusqu'au XIXe siècle. Par extension, le terme s'applique aux peuples soumis à une domination étrangère ou à un gouvernement despotique. Au sens figuré, il désigne l'état de ceux qui sont

3. Sauf dans le cas de la catachrèse où, par définition, il n'existe pas de terme approprié. Mais cette exception présuppose que la substitution remplace nécessairement un terme par un autre. Or si, dans la catachrèse «le pied de la montage», il n'y a effectivement pas de vocable isolé substituable à "pied», le syntagme "partie inférieure » pourrait jouer ce rôle; en ce sens, la substitution serait toujours possible. 
soumis à une autorité tyrannique, qui se trouvent sous la dépendance d'une autre personne. Ou encore ce qui assujettit, ce qui subjugue : être esclave d'un sentiment, d'un principe. Ou enfin ce qui laisse peu de liberté, peu de loisir. Le noyau sémantique commun à ces cinq acceptions, c'est l'absence de liberté, la domination. Dans les emplois non figurés du mot esclavage, la domination est le résultat d'une contrainte sociale globale s'appliquant soit à des individus, soit à des collectivités. Dans les emplois figurés, la domination s'exerce sur des individus qui semblent y consentir en se soumettant soit à une personne, soit à des éléments de leur propre existence: sentiment ou principe dans un cas, activité dans l'autre. C'est sur cette toile de fond sémantique qu'il faut lire les emplois heuristiques de la métaphore de l'esclavage dans les textes féministes. Nous en examinerons deux exemples : celui du mariage interprété comme une institution esclavagiste, et celui du «sexage ${ }^{4}$.

\subsection{Le mariage selon Sheila Cronan (1971)}

Cronan veut montrer que le mariage constitue une forme d'esclavage. L'esclave, pour elle, c'est une personne possédée par une autre personne, contrainte de travailler sans salaire pour son propriétaire et de lui obéir. Les trois traits heuristiques de cette définition sont donc la possession d'un être humain, le travail gratuit et l'obéissance.

De prime abord, le premier trait semble inapplicable: ne peut-on faire valoir, en effet, que contrairement aux esclaves, les femmes non seulement ne sont pas vendues, mais encore s'engagent librement dans le mariage en signant formellement un contrat à l'avantage des deux parties?

La stratégie de Cronan consiste à nier que le mariage soit un véritable contrat. Car il s'agit du seul «contrat» légal important dont les termes ne sont pas énumérés: une farce donnant aux

4. Les dispositions légales auxquelles se réfèrent ces deux exemples sont évidemment relatives à l'époque et au lieu de production des textes présentés : les États-Unis de 1970 dans le cas de Cronan, la France de 1978 dans celui de Guillaumin. Que ces dispositions ne coïncident pas toutes avec la situation actuelle au Québec n'affecte pas l'exemplarité de ces textes. 
femmes "l'illusion qu'elles consentent à une relation mutuellement bénéfique, alors qu'en fait elles signent leur acte d'esclavage». Comment peuvent-elles se laisser piéger ainsi? Parce qu'elles ont intériorisé, depuis des milliers d'années, un rôle qu'elles en sont venues à croire naturel; comme l'a souligné par ailleurs Andrée Michel (1975: 31), dans la première étape de ses rapports avec l'oppresseur, l'opprimé, à cause de son isolement, s'identifie à lui et adopte son idéologie. Second motif : la société n'offre aux femmes aucune autre véritable carrière que le mariage, car si elles tentent de vivre par leurs propres moyens, elles sont en butte, entre autres, à la discrimination des employeurs, à l'opprobre social, à la crainte perpétuelle d'être attaquées et à l'exploitation sexuelle. En troisième lieu, les femmes sont trompées sur la véritable nature du mariage, dont on leur fait croire qu'il est à leur avantage, qu'il les protège : une " protection » analogue à celle de l'esclavage censé «protéger » les Noirs. Enfin, l'idéologie maquille de façon tellement attirante et romantique le mariage que les jeunes filles s'y précipitent sans savoir ce qui les attend réellement. L'absence de vente effective, dont on sait par ailleurs qu'elle caractérise, sous le mode de l'échange, d'autres types de sociétés, n'empêche donc pas que les femmes soient réellement possédées par les hommes qu'elles épousent.

Les femmes ont peut-être aussi l'illusion que c'est librement qu'elles s'adonnent au travail domestique; mais les cours de justice américaines en ont décidé autrement, en stipulant non seulement que le mari a un droit légal au travail de son épouse, mais encore que celle-ci ne peut être rémunérée pour cette fonction. À noter d'ailleurs la disparité des responsabilités réciproques: alors que l'épouse doit fournir tous les services domestiques nécessaires, c'est-à-dire entretenir la maison, satisfaire les besoins personnels du mari et s'occuper des enfants, l'époux n'est astreint à lui fournir que le lit et la nourriture. Et si jamais il n'y parvient pas, l'épouse n'a d'autre recours que de cumuler travail extérieur et travail domestique. Etre une épouse est donc un emploi à temps plein mais non rémunéré : n'est-ce pas cela, l'esclavages?

5. L'esclavage, ici, c'est le travail forcé. On voit par cet exemple comment la même métaphore peut être employée récursivement pour chacun de ses traits pertinents. Si 
Dernier trait pertinent: l'obéissance. Celle-ci s'exerce d'abord sur le plan de la sexualité. Parce que mari et femme se promettent un amour mutuel lors de la cérémonie nuptiale, on a tendance à croire que l'amour est le but du mariage. Mais, en cas de divorce, l'amour n'est pas un facteur légalement pertinent. Par contre, la sexualité, pudiquement passée sous silence lors de la cérémonie, est, d'après la loi, impliquée dans la notion même de mariage: s'y refuser est une cause d'annulation pour fraude. Par ailleurs, la prestation sexuelle étant considérée comme obligatoire, le mari peut contraindre son épouse à se plier à ses désirs sans être accusable de viol. Même si elle n'est pas toujours mise à exécution, la menace de l'usage de la force pèse donc constamment sur les rapports conjugaux. De plus, la contrainte sexuelle est à l'avantage de l'époux, qui y trouve toujours son compte, alors que l'orgasme féminin est plus aléatoire.

En dépit de l'accent qui a été mis récemment sur le devoir de l'époux de procurer du plaisir à son épouse, cela ne se produit pas la plupart du temps, et nous savons toutes qu'une relation sexuelle sans orgasme est au mieux une perte de temps. Les statistiques sus-mentionnées nous montrent que 70 pour cent des épouses américaines subissent cette expérience ennuyeuse et souvent douloureuse plus de deux fois sur trois. (Cronan 1971: 63)

L'obéissance qui caractérise l'esclavage s'exerce aussi dans l'absence de liberté de mouvement de l'épouse. Le mari a le droit de décider du lieu de résidence du couple et, s'il doit déménager, son épouse est obligée de le suivre. En cas de refus, il peut l'accuser de désertion. Et là où la désertion est un motif de divorce, l'épouse sera considérée comme la partie «en faute», et privée de pension alimentaire.

Légalement possédée par son mari, forcée de travailler pour lui sans rémunération et soumise à ses volontés, l'épouse serait donc l'esclave de l'époux. Or cet assujettissement d'une personne à une autre découle en fait, selon Colette Guillaumin, d'une appropriation collective : le sexage.

le texte ne pratique pas lui-même ce jeu, c'est à l'interprète d'essayer les divers sens possibles. 


\subsection{Le sexage selon Colette Guillaumin (1978A et B)}

Guillaumin stipule que la forme spécifique de l'oppression des femmes est leur exploitation, en tant que classe, par les hommes, l'idée de nature constituant l'expression idéologique de cette appropriation qui, en tant que telle, relève de l'esclavage:

Ce qui nous concernera ici est l'appropriation physique elle-même, le rapport où c'est l'unité matérielle productrice de force de travail qui est prise en mains, et non la seule force de travail. Nommé « esclavage » et «servage » dans l'économie foncière, ce type de rapport pourrait être désigné sous le terme "sexage» pour ce qui concerne l'économie domestique moderne, lorsqu'il concerne les rapports de classes de sexe. $(A: 9)$

Le sexage est à l'économie domestique moderne ce que l'esclavage et le servage furent à l'économie foncière. Cette proportion autorise sans doute une métaphore analogique de type classique: le sexage est l'esclavage d'un sexe par rapport à l'autre. Mais s'en tenir à ce niveau, ce serait occulter la radicalité du texte de Guillaumin, qui nous invite à reconnaître, derrière les fluctuations terminologiques liées au cours de l'histoire, et derrière le passage d'un type d'économie à l'autre, une même structure d'oppression. En ce sens, l'esclavage au sens littéral, le servage et le sexage appartiennent à un même ensemble que, par catachrèse ${ }^{6}$ et selon le parcours de l'espèce au genre, on pourrait aussi nommer "esclavage».

Les deux traits heuristiques de cette métaphore et de la structure à laquelle elle renvoie sont l'appropriation de la force de travail et l'appropriation physique du travailleur ou de la travailleuse: voyons-les à l'œuvre dans le sexage.

Économiquement parlant, les femmes sont doublement exploitées: moins bien payées que les hommes si elles travaillent à l'extérieur du foyer, elles accomplissent gratuitement le travail domestique. Si la force de travail est l'ultime chose que l'ouvrier

6. La « nomination catachrérique » constitue, selon Pierre Marchal (1980: 107), la forme de métaphore que privilégie le discours scientifique. La majeure partie des termes scientifiques a une origine figurée, renchérit Jean Molino (1979A : 86; 1979B:103), qui précise toutefois que si, dans les sciences de la nature, la métaphore devient modèle explicatif, ce processus d'épuration est impossible en sciences humaines, où "la métaphore originaire demeure largement métaphorique». 
puisse vendre pour vivre, force est d'admettre que la classe entière des femmes est dépourvue de ce privilège : elle subit non seulement l'accaparement de sa force de travail, mais un rapport d'appropriation physique direct. Concrètement, le sexage s'exprime dans l'appropriation du temps des femmes, qui n'est pas mesuré dans le contrat de mariage; dans l'appropriation des produits du corps, puisqu'il n'y a pas de limite contractuelle au nombre d'enfants, et que ceux-ci, d'après la législation française, appartenaient au père; dans l'entretien physique des membres du groupe familial, qui incombe toujours aux femmes; et dans l'obligation sexuelle, qui est un usage physique de la femme. Il existe, selon Guillaumin, deux formes principales de l'usage physique sexuel: par contrat non monétaire dans le mariage, et par prestation monnayée dans la prostitution. Superficiellement opposées, ces deux formes expriment conjointement l'appropriation de la classe des femmes, la présence d'un paiement dans la seconde n'ayant pour effet que de limiter l'usage physique à l'usage sexuel et d'en mesurer le temps. Dans le mariage, par contre, l'usage physique est illimité, mais sa dimension sexuelle est obligatoire et cause d'annulation en cas de non-exercice. Par ailleurs, que l'adultère soit en général toléré pour l'homme, mais cause de divorce dans le cas de la femme, cela montre bien que celle-ci est la propriété de son époux et ne peut disposer de son propre corps.

Mais ne pourrait-on objecter, comme dans le cas du texte de Cronan, que les femmes acceptent librement cette situation en signant le contrat de mariage ? L'absence de mesure monétaire ou temporelle, jointe à l'absence de salaire, montre, selon Guillaumin, que les femmes ne vendent pas leur force de travail, qu'elles sont acquises une fois pour toutes, et ce, sans contrat. Le mariage, en effet, constitue la forme institutionnelle individualisée du sexage, qu'il entérine et légalise par un contrat dont la femme est exclue. Elle en est exclue d'abord parce qu'elle ne cède pas de force de travail :

Le fait qu'il n'y ait pas de terme au travail, pas de mesure de temps, pas de notion de viol [...], montre que cette cession est faite en bloc et sans limites. Et que, par conséquent, ce qui est cédé n'est pas la force de travail mais bien l'unité matérielle que forme l'individu lui-même. (A: 20). 
Déjà cédée en bloc, la femme ne s'appartient plus, et par conséquent elle n'est pas partie prenante au contrat de mariage :

Pour contracter, la propriété de biens matériels (fonciers et monétaires mis en jeu dans le contrat), éventuellement la propriété de choses vivantes (animaux, esclaves, femmes, enfants...) semble superficiellement déterminante, mais ce qui est déterminant est la propriété de soi-même, qui s'exprime, à défaut de tout «bien propre », dans la possibilité de vendre sa propre force de travail. Telle est la condition minimale de n'importe quel contrat. Or le fait pour l'individu d'être la propriété matérielle d'autrui l'exclut de l'univers du contrat ; on ne peut pas être à la fois propriétaire de soi-même et être la propriété matérielle d'autrui $(A: 20)$.

Que cette appropriation physique des femmes relève de la contrainte, et non du libre choix, cela se voit à ses moyens. Tout d'abord, ne donnant pas assez d'argent aux femmes pour qu'elles assurent leur existence et celle de leurs enfants, le marché du travail les contraint à se vendre comme épouses. En second lieu, les femmes sont confinées spatialement, car c'est le mari qui fixe le domicile. Ensuite, la violence physique constitue la sanction socialisée du droit que s'arrogent les hommes sur les femmes. De plus, « la contrainte sexuelle sous forme de viol, de provocation, de drague, d'épuisement, etc. est, d'abord, l'un des moyens de coercition employé par la classe des hommes pour soumettre et apeurer la classe des femmes, en même temps que l'expression de leur droit de propriété sur cette même classe» (A : 25); c'est pourquoi, d'ailleurs, la violence sexuelle envers une femme n'est considérée comme viol « que si elle est susceptible de produire des enfants à un bomme non consentant [...], que si le propriétaire de la femme (mari ou père), donc des enfants de la femme, risque de se retrouver avec des enfants non propres » $(A: 26)$. Enfin, dernier moyen, c'est l'arsenal juridique qui fixe les modalités de l'appropriation des femmes, car celles-ci ne sont pas considérées comme sujets juridiques: elles appartiennent à leurs maris qui n'ont d'autre obligation à leur égard que de les entretenir, c'est-àdire, comme le notait Cronan, de leur fournir le lit et la nourriture.

\subsection{La métaphore argumentative}

Dans leur Traité de l'argumentation (1976: 192; 499-549) Perelman et Olbrechts-Tyteca ont fortement insisté sur le rôle 
argumentatif des figures en général, et en particulier sur celui de l'analogie et de la métaphore. L'analogie, dont la métaphore constitue une condensation, est un raisonnement portant sur des relations. Étant donné quatre termes, le rapport entre les deux premiers, qui constituent le thème, est semblable au rapport entre les deux derniers, qui composent le phore. Le but de cette assimilation est d'éclairer, de structurer, d'évaluer le thème grâce à ce qu'on sait du phore, lequel appartient à un domaine hétérogène censément mieux connu. Le maniement d'un tel instrument est délicat. Dès qu'on développe l'analogie ou la métaphore, leur rôle heuristique se dissocie en effet de leur fonction probante, une tension s'instaurant entre le désir de prolonger indéfiniment l'analogie pour voir où cela mènera, et la nécessité de ne pas dépasser la limite au-delà de laquelle la conviction n'est plus possible, et où le destinaire récalcitrant n'y veut plus voir qu'une figure de style.

Expressément ou implicitement, Cronan posait que le mariage est un esclavage, et Guillaumin affirmait que la classe des femmes est esclave de celle des hommes. Dans l'un et l'autre texte, les caractéristiques de l'esclavage sont à peu près les mêmes: appartenance à une autre personne, travail non rémunéré, obéissance caractérisée, entre autres, par la contrainte sexuelle et la résidence forcée. Dans les deux cas le caractère illusoire du contrat de mariage est dénoncé, de même que le sont la violence masculine et la coercition sociale au mariage. La différence majeure entre les deux auteures réside dans la notion de sexage, introduite par Guillaumin pour désigner l'esclavage collectif de la classe des femmes. Si l'on s'en tient à l'esclavage individuel de l'épouse, il est évident qu'il ne s'agit, ici, ni d'un peuple soumis à la domination ou au despotisme, ni de l'assujettissement à une passion ou à une simple activité qui ne laisse guère de loisir. Il ne peut s'agir non plus du sens littéral, puisque les esclaves de l'Antiquité, tout comme les Noirs plus tard, étaient reconnus comme esclaves, et d'abord par leur maîtres, ce qui n'est pas le cas des femmes. Reste donc le sens figuré en vertu duquel une personne est soumise à l'autorité tyrannique d'une autre personne et se trouve sous sa dépendance. Dépendance choisie, avons-nous suggéré. Mais tant le texte de Cronan que celui de Guillaumin montrent que les femmes ne choisissent pas vraiment leur sort: 
c'est la société qui les contraint au mariage, et le contrat qu'elles signent n'est qu'une mascarade. En ce sens, tout comme l'esclave antique ou le Noir des plantations de coton, la femme mariée se trouverait, contre son gré, sous la puissance absolue d'un maître, elle serait esclave en un sens quasi littéral.

Qui n'accepte pas cette conclusion pourra, s'il ne s'enferme pas dans l'arrogance de la simple fin de non-recevoir, argumenter à partir des traits heuristiques de la métaphore initiale ou des éléments de la définition du mot dans son acception «littérale»: je me contenterai d'indiquer quelques orientations de ces prolongements dialogiques de notre métaphore heuristique. Le contredicteur, par exemple, reprochera à Cronan de réduire la sexualité à l'orgasme, et celui-ci à des relevés statistiques qui prouvent déjà, pourtant, que certaines femmes au moins tirent bénéfice du commerce sexuel et donc que celui-ci n'est pas à l'avantage exclusif des hommes. Il objectera que la supposée menace constante de la violence est une fiction qui fait abstraction de ce que ressentent réellement les personnes. Ou que le véritable esclave devait exécuter n'importe quel travail que lui confiait son maitre, ce qui n'est pas le cas de la femme mariée. Ou que l'assimilation du mariage à la prostitution, que suggère Guillaumin, est excessive. Ou que le véritable maître a droit de vie et de mort sur l'esclave, ce qui n'est pas le cas du mari. De telles objections devraient être étayées et permettre une riposte menant soit à leur réfutation, soit au nuancement réciproque des positions. Dans l'un comme dans l'autre cas, il devrait être évident que la métaphore initiale a permis une repensée radicale du mariage, repensée dont la limite est la redéfinition conceptuelle du sinistre domaine de la domination de l'être humain par un autre être humain. La théorie de la métaphore aura ainsi permis de comprendre comment telle métaphore particulière, simple cliché du point de vue de la fonction ornementale, peut jouer un rôle d'autant plus stratégique sur le plan théorique qu'elle en appelle, au-delà de la pure connaissance, à la reconnaissance de la nécessité d'une action révolutionnaire au niveau de la théorie elle-même. 


\section{DE LA THÉORIE DE LA MÉTAPHORE À LA MÉTAPHORE DE LA THÉORIE}

Implicitement ou expressément, toute définition du féminisme s'articule en trois segments: un élément générique posant qu'il s'agit d'une théorie et/ou d'une pratique, individuelle et/ou collective, de femmes ou de femmes et d'hommes; un trait spécifique négatif dénonçant la domination masculine en l'associant ou pas aux problèmes posés par le racisme et la division de la société en classes; et un facteur spécifique positif proposant une métamorphose réformiste ou révolutionnaire de cette situation d'oppression 7 . L'importance stratégique de la métaphore de l'esclavage tient à ce qu'elle symbolise le trait spécifique négatif de la domination masculine. Or accepter de considérer cette métaphore comme un concept littéral, cela permet aussi de l'insérer dans un argument par l'exemple qui, à son tour, annoncera symboliquement le facteur spécifique positif du féminisme.

Revenons au Traité de l'argumentation de Perelman et Olbrechts-Tyteca (1976:502;474):

Lorsque les deux rapports que l'on confronte appartiennent à un même domaine, et peuvent être subsumés sous une structure commune, l'analogie fait place à un raisonnement par l'exemple ou l'illustration, thème et phore fournissant deux cas particuliers d'une même règle.

L'emploi de l'argumentation par l'exemple, bien qu'ouvertement proclamé, tend souvent à nous faire passer de celui-ci à une conclusion également particulière, sans qu'aucune règle soit énoncée: c'est ce qu'on appelle l'argumentation du particulier au particulier.

De ce particulier que fut l'esclavage au sens littéral, à ces particulières que sont les femmes, voici le passage qu'effectue TiGrace Atkinson (1975 : 21):

Le mariage et la famille sont des institutions corrompues comme le fut l'esclavage. Ils doivent être abolis comme le fut l'esclavage. Par définition ils oppriment et exploitent nécessairement les groupes qui leur sont soumis. Si les femmes étaient libres, libres de grandir comme des personnes, libres de se créer elles-mêmes, libres d'aller où elles

7. La mise en rapport de ces divers éléments incire à considérer « le» féminisme non comme une essence simple qu'une définition pourrait maîtriser, mais comme un champ sémantique, idéologique et pragmatique parcouru d'axes majeurs et d'orientations plus spécifiques : cf. Bouchard 1986. 
veulent, libres dêtre où elles veulent, libres de choisir leurs vies, il n'y aurait pas d'institutions comme le mariage ou la famille. Si les esclaves avaient eu ces libertés, il n'y aurait pas eu d'esclavage.

«Il n'y aurait pas eu d'esclavage»: la formulation au passé de cette proposition est peut-être son trait le plus important. Il y a eu des esclaves. Mais les esclaves ont été ou se sont libérés. Assimiler les femmes à des esclaves, c'est aussi leur faire entendre le chant de la libération possible. La métaphore de l'esclavage est une métaphore espérante. Conjurant une société différente où l'esclavage sous toutes ses formes serait définitivement aboli, elle est d'essence hétéropolitique.

Par hétéropolitique, j'entends l'élaboration discursive d'un modèle de société idéalisée, élaboration éventuellement suivie d'un relais idéologique préoccupé de ses possibilités de réalisation et d'une implantation historique sous forme de commune, d'institution parallèle, etc. Ce moment discursif de la démarche hétéropolitique se réalise soit dans une fiction (l'utopie proprement dite), soit dans un texte théorique (la para-utopie, qui est tantôt laïque et tantôt, comme dans les messianismesmillénarismes, d'inspiration religieuse). L'Utopie de Thomas More, la République de Platon et l'odyssée des Shakers en terre américaine illustrent ces trois facettes de la discursivité hétéropolitique ${ }^{8}$.

Entre le discours hétéropolitique et son éventuelle implantation historique s'intercale la médiation idéologique, qui oscille de la simple disposition à l'action engendrée par l'utopie?, aux stratégies et tactiques nécessaires à la réalisation d'un modèle para-utopique ou millénariste. Or l'omniprésence de la métaphore de l'esclavage dans les textes féministes est l'indice de la pertinence d'une lecture hétéropolitique de ces textes. Sur le plan des différents types de discours, cette lecture nous enseigne que nous n'avons jamais connu que des métaphores de théorie. Pour le montrer, une classification des principaux types de discours s'impose.

8. Sur cette notion, cf. Bouchard 1985A et 1985B.

9. L'utopie n'est pas un modèle à réaliser. En plus de sa fonction esthétique, qu'elle partage avec toutes les formes d'art, elle a pour rôle principal d'éveiller la conscience politique des lecteurs en contestant le réel au nom d'un possible présenté comme déjà existant. 
Les signes fonctionnent soit isolément, comme la canne blanche de l'aveugle, soit grâce à un ensemble, à une sémie. Les sémies sont asystématiques, tel le cas des marques d'appréciation du public au spectacle, ou systématiques. La principale sémie systématique est le langage, parlé ou écrit. En deçà de la phrase, linguistique et grammatologie démontent des systèmes. Au-delà de la phrase, la sémiotique discursive analyse les divers types de discours: le discours libre, c'est-à-dire la conversation, mais surtout les discours réglès soumis à une sur-systématisation spécifique. Ces discours réglés sont le discours idéique, qu'il soit démonstratif ou argumentatif ${ }^{10}$; le discours esthétique, tantôt poétique et tantôt narratif ; le discours pragmatique qui, sous la forme du sermon, de la harangue politique, de la plaidoierie juridique ou du message publicitaire, incite directement le destinataire à l'action; et le discours féministe. Schématiquement : ${ }^{11}$

10. Est démonstratif le discours de la science au sens le plus rigoureux du terme. Relèvent de l'argumentation les sciences humaines, la philosophie et la théologie.

11. Ce schéma ne prétend pas à l'exhaustivité. Par ailleurs, la trilogie du discours idéique, du discours esthétique et du discours praginatique correspond aux fonctions cognitive (référentielle), poétique et conative du schéma jakobsonien de la communication. 
(SÉMIOTIQUE)

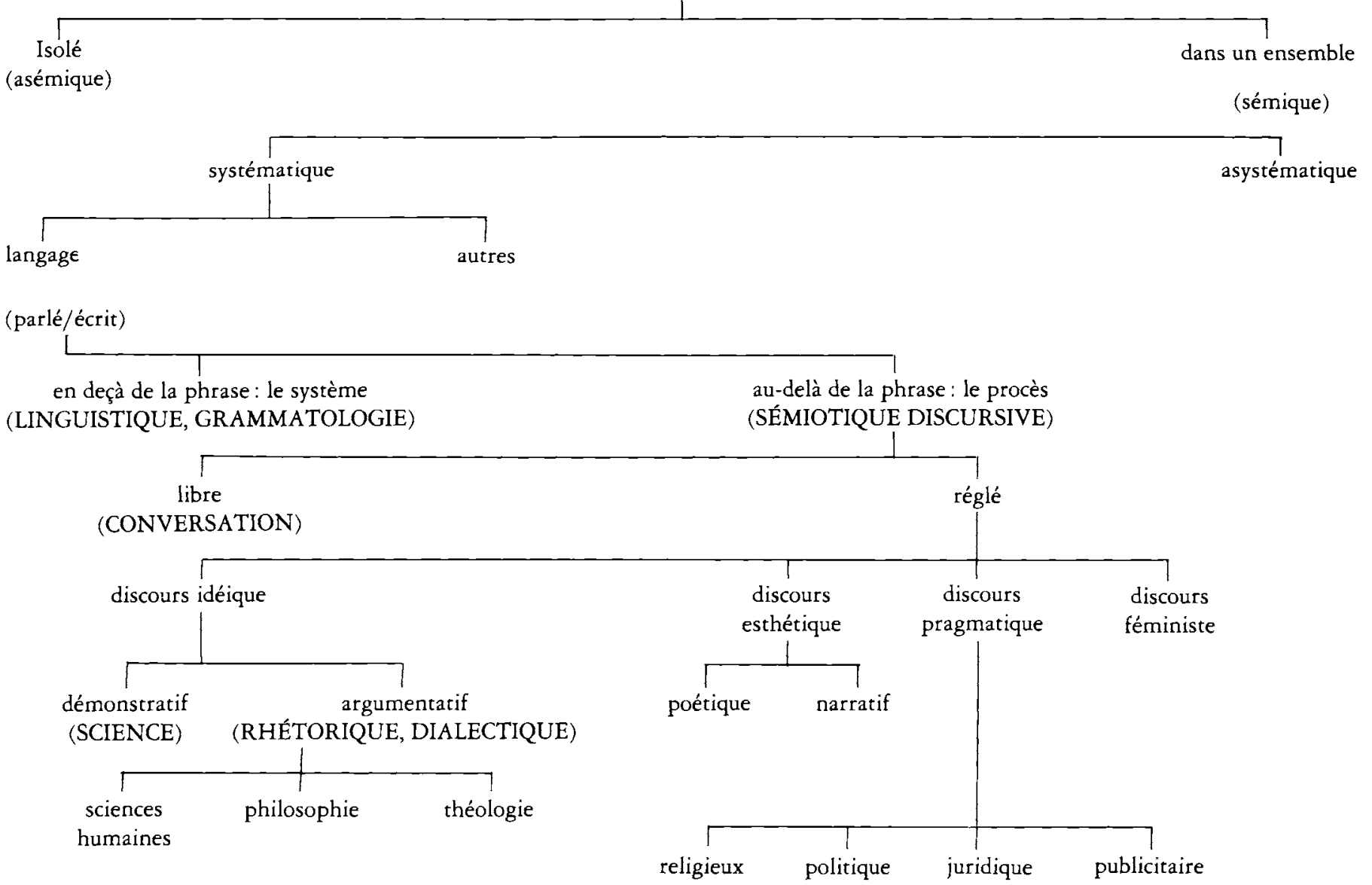


Le discours féministe ne peut-il être idéique, esthétique ou pragmatique? N'existe-t-il aucun poème, aucun roman, aucun texte philosophique féministes? Du strict point de vue des principes d'une bonne classification, cette typologie est déficiente. Déficience volontaire. Et subversive. Elle suggère que tous les autres types de discours, loin d'être sexualement neutres, ont en fait été, dans l'immense majorité des cas, non seulement masculins, mais masculinistes : véhicules occultes, et d'autant plus pervers, des valeurs des hommes, caution idéologique de l'oppression des femmes. Même dans ce noyau de la pensée abstraite que l'on croyait immunisé à l'infiltration des valeurs sociales : l'épistémologie, la métaphysique, la méthodologie et la philosophie des sciences, des préjugés sexuaux ont toujours infléchi le savoir et la recherche dans le non-sens de la domination masculine ${ }^{12}$. Si la théorie se doit d'être neutre et universelle, nous n'avons donc jamais connu que des métaphores de théories. La théorie a toujours été en esclavage. Comment la libérer? Si l'on considère la théorie masculine comme une métaphore du genre pour l'espèce, trois parcours sont possibles : de l'espèce à une autre espèce, de l'espèce au genre, de l'espèce à l'analogue. De l'espèce à une autre espèce: substituer à la théorie masculine une théorie spécifiquement féminine, un matriarcat de la pensée. Ce qui, sans doute, ne saurait advenir sans une transformation de la société dans son ensemble. Illustrent cette transformation les utopies féministes qui, comme The Female Man de Joanna Russ (1975) ou Les guérillères de Monique Wittig (1969), racontent l'élimination physique des hommes ou leur soumission à la nouvelle loi. De l'espèce au genre : remplacer la théorie masculine par une théorie enfin neutre, enfin universelle. C'est-à-dire, sur le plan sociopolitique, instaurer une société sans domination sexuale, authentiquement égalitaire, comme celle que suggère Ursula Le Guin dans The Dispossessed (1975). De l'espèce à l'analogue: disqualifier la théorie, parce qu'elle est incurablement masculine, au profit d'une nouvelle pratique discursive, d'une hétérosophie par rapport à laquelle la philosophie, la théorie, le féminisme même seraient dépassés ${ }^{13}$. C'est-à-dire tenter non plus d'inverser

12. Cf. Harding et Hintikka 1983. Ce collectif fournit plusieurs pistes de recherches, bibliographiques entre autres, en liaison avec cette problématique.

13. CE. Braidorti 1983. 
le rapport de domination entre les sexes, ni même de le neutraliser, mais de le rendre impensable en concevant par exemple, comme Ursula Le Guin (1969) dans The Left Hand of Darkness, un monde androgyne ${ }^{14}$. L'androgynie biologique est évidemment une solution purement romanesque, mais elle fonctionne comme métaphore d'une androgynie comportementale où nulle activité sociale n'est attribuée à un(e) individu(e) sur la base de son appartenance sexuale:

Quand vous rencontrez un Géthénien, vous ne pouvez ni ne devez faire ce que fait naturellement un être d'une espèce bisexuée, l'insérer dans le rôle d'Homme ou de Femme, tout en adoptant le rôle correspondant selon vos attentes des interactions prévues ou possibles entre personnes du même sexe ou de sexe opposé. Toute notre structure d'interaction socio-sexuelle est ici inexistante. Ils ne peuvent jouer à ce jeu. Ils en se voient pas les uns les autres en tant qu'hommes ou femmes. Il est presque impossible à notre imagination d'accepter cela. (LHD : 68-69)

\section{OUVERTURE}

Partis d'une métaphore spécifique, celle de l'esclavage dans les textes féministes, nous avons montré comment, de façon heuristique, elle nous invite à modifier les rapports entre les sexes et débouche ainsi sur une vision hétéropolitique de la société. Mais si cette vision féministe remet globalement en cause non seulement les théories politiques antérieures mais aussi les conceptions hétéropolitiques qui, depuis Aristote jusquaux projets contemporains de sociétés idéales, ont tenté de repenser les rapports sociaux sans redéfinir les relations entre les sexes, elle nous oblige aussi à réévaluer nos conceptions du théorique en tant que tel et à les réarticuler par rapport aux projets sociaux qu'elles sous-tendent et reflètent : c'est pourquoi il nous a fallu conjuguer les métamorphoses possibles de la théorie et celles de la société dans une même aspiration totale à la libération des êtres humains.

Faculté de philosopbie, Université Laval.

14. Au colloque L'esthétique aujourd'hui, tenu au Cegep de Ste-Foy le 7 novembre 1986, ¡’ai présenté, sous le titre «Esthétique et féminisme : le cas Le Guin», une étude de ce roman qui paraîtra dans les Actes du colloque. 


\section{BIBLIOGRAPHIE}

ARISTOTE, (1962): La politique (trad. Tricot), Paris : Vrin.

ARISTOTE, (1961) : Poétique (trad. Hardy), Paris : Les Belles Lettres.

ATKINSON, Ti-Grace, (1975): Odyssée d'une Amazone, Paris: Éditions des femmes.

BEAUVOIR, Simone de, (1968 A et B): Le deuxième sexe (2 vol.), Paris: Gallimard, Coll. Idées n. 152 et 153.

BEBEL, Auguste, (1979) : La femme dans le passé, le présent et l'avenir, Genève : Slatkine Reprints, Coll. Ressources.

BLACK, Max, (1979) : «La métaphore», Poésie, 5, p. 92-107.

BOUCHARD, Guy, (1984) : Le procès de la métaphore, Montréal : Hurtubise $\mathrm{HMH}$.

BOUCHARD, Guy, GIROUX, Laurent, LECLERC, Gilbert, (1985) : L'utopie aujourd'bui, Montréal : Presses de l'Université de Montréal.

BOUCHARD, GUY, (1985 A) : «Eutopie, dystopie, para-utopie et péri-utopie », in BOUCHARD \& GIROUX \& LECLERC, (1985).

BOUCHARD, Guy, (1985 B) : «L'hétéropolitique de l'histoire », in BOUCHARD \& GIROUX \& LECLERC, (1985).

BOUCHARD, Guy, (1986) : «Féminisme et philosophie : jalons », Considérations, 7, 2/3, p. $23-63$.

BRAIDOTTI, Rosi, (1983) : «Femmes et philosophie : questions à suivre... », $L a$ revue d'en face, 13, p. 23-33.

CRONAN, Sheila, (1971) : «Marriage», in Koedt \& Firestone, (1971), p. 62-65.

ENGELS, Friedrich, (1966) : L'origine de la famille, de la propriété privée et de l'État, Paris : Éditions sociales.

FONTANIER, Pierre, (1968): Les figures du discours, Paris : Flammarion.

GUILlAUMIN, Colette, (1978): «Pratique du pouvoir et idée de Nature: (A) L'appropriation des femmes (B) Le discours de la nature», Questions féministes 2, p. 5-27; 3, p. 5-28.

HARDING, Sandra, HINTIKKA, Merrill, éd., (1983): Discovering Reality. Feminist Perspectives on Epistemology, Metaphysics, Methodology, and Philosophy of Science, Dordrecht \& Boston \& London : D. Reidel.

KOEDT, Anne, FIRESTONE, Shulamith, éd., (1971) : Notes From the Third Year, New York: Notes From the Second Year, Inc.

LE GUIN, Ursula, K., (1969) : The Left Hand of Darkness, New York : Walker and $\mathrm{Co}$

LE GUIN, Ursula, K., (1975) : The Dispossessed, New York: Avon Books.

MARCHAL, Pierre, (1980) : «Discours scientifique et déplacement métaphorique », La métaphore (éd. : René JoNGEN), Bruxelles: Facultés universitaires Saint-Louis. 
MICHAUD, Stéphane, (1984) : Flora Tristan (1803-1844), Paris : Les éditions ouvrières.

MICHEL, Andrée, (1975): «Naissance d'une conscience féministe », in L'Arc, 61, p. 31-38.

MILL, John Stuart, (1975): L'asservissement des femmes, Paris: Petite bibliothèque Payot, n. 254.

MOLINO, Jean, (1969A): "Métaphores, modèles et analogies dans les sciences ", Langages, 54, p. 83-102.

MOLINO, Jean, (1979B) : "Anthropologie et métaphore», Langages, 54, p. $103-125$.

PERELMAN, Ch., OLBRECHTS-TYTECA, L., Traité de l'argumentation. La nouvelle rhétorique, Bruxelles: Éditions de l'Université de Bruxelles, 1976.

RICOEUR, Paul, (1975) : La métaphore vive, Paris : Seuil.

RUSS, Joanna, (1975): The Female Man, New York: Bantam.

WITTIG, Monique, (1969) : Les guérillères, Paris : Éd. de Minuit.

WOLLSTONECRAFT, Mary, (1976) : Défense des droits de la femme, Paris:

Petite bibliothèque Payot. 